\title{
Visual marking and the perception of salience in visual search
}

\author{
BRADLEY S. GIBSON \\ University of Notre Dame, Notre Dame, Indiana \\ and \\ YUHONG JIANG \\ Yale University, New Haven, Connecticut
}

\begin{abstract}
In the present study, the gap paradigm originally developed by Watson and Humphreys (1997) was used to investigate whether the process of visual marking can influence the perceptual salience of a target in visual search. Consistent with previous studies (Watson \& Humphreys, 1997), the results showed that search was not affected by the presence of the preceding distractors when the target was relatively low in salience. This finding suggests that visual marking can increase the efficiency of visual search by decreasing the size of the search set. However, more important, the results also showed that search was affected by the presence of the preceding distractors when the target was relatively high in salience. This finding suggests that visual marking may be limited in its ability to increase the perceptual salience of the target. Together, the results of the present study suggest that the effectiveness of visual marking may vary as a function of search context.
\end{abstract}

Visual search often relies on a variety of mechanisms that serve to prioritize the order in which currently available objects are selected for further processing. In many natural contexts, however, the set of searchable objects may not be available at the same time. Rather, the set of searchable objects may change over time, with some objects appearing later than others. In such cases, visual search may also rely on mechanisms that serve to deprioritize, or inhibit, preexisting objects that are known to be irrelevant. One such inhibitory mechanism has come to be called visual marking (Watson \& Humphreys, 1997).

Initial evidence for visual marking was provided by comparing performance in a modified conjunction search, or gap, condition with performance in two baseline conditions (Watson \& Humphreys, 1997). The all-element baseline condition involved a conjunction search task in which observers searched for the presence of a blue $H$ target that could appear among several blue $A$ and green $H$ distractors. In contrast, the half-element baseline condition involved a search task in which only the blue elements from the all-element baseline condition were shown. In this half-element baseline condition, observers once again searched for the presence of a blue $H$ target, but now the target (when present) only appeared among blue $A$ distractors. Both of these baseline conditions can be con-

The research was supported by Grant SBR-9817245 from the National Science Foundation awarded to B.S.G. The authors thank Art Kramer, Derrick Watson, and Jeremy Wolfe for providing valuable comments on previous versions of this article. Correspondence concerning this article should be addressed to B. S. Gibson, Department of Psychology, 118 Haggar Hall, University of Notre Dame, Notre Dame, IN 46556 (e-mail: gibson.16@nd.edu). trasted with the gap condition. Like the all-element baseline condition, the task in the gap condition again required searching for the presence of a blue $H$ target that could appear among both blue $A$ and green $H$ distractors. However, in the gap condition, there was a 1,000-msec gap inserted between the appearance of the green $H$ distractors and the appearance of the blue letters. In other words, the green $H$ distractors appeared $1,000 \mathrm{msec}$ before the critical display of blue letters. In each condition, visual search efficiency was evaluated by measuring the extent to which search time was affected by the number of display elements (which varied from as few as 4 elements to as many as 16 elements). ${ }^{1}$ A representative example of these three types of displays is shown in Figure 1.

The results of Watson and Humphreys's (1997) experiments showed that visual search was performed twice as efficiently in the gap condition as it was in the all-element baseline condition, suggesting that only half of the display elements were searched in the gap condition; in fact, visual search was performed just as efficiently in the gap condition as it was in the half-element baseline condition (see also Theeuwes, Kramer, \& Atchley, 1998). On the basis of this and other evidence, Watson and Humphreys concluded that the locations occupied by the preceding green $H$ distractors could be marked as irrelevant via inhibition and therefore excluded from consideration when the critical target display appeared. As such, these findings have important theoretical implications for current theories of visual search (see Wolfe, 1998, for a recent review) and suggest that a form of memory-based, inhibitory tagging can operate to improve the efficiency of visual search by removing old distractors from the search set, at least when these distractors are presented in advance of the critical 


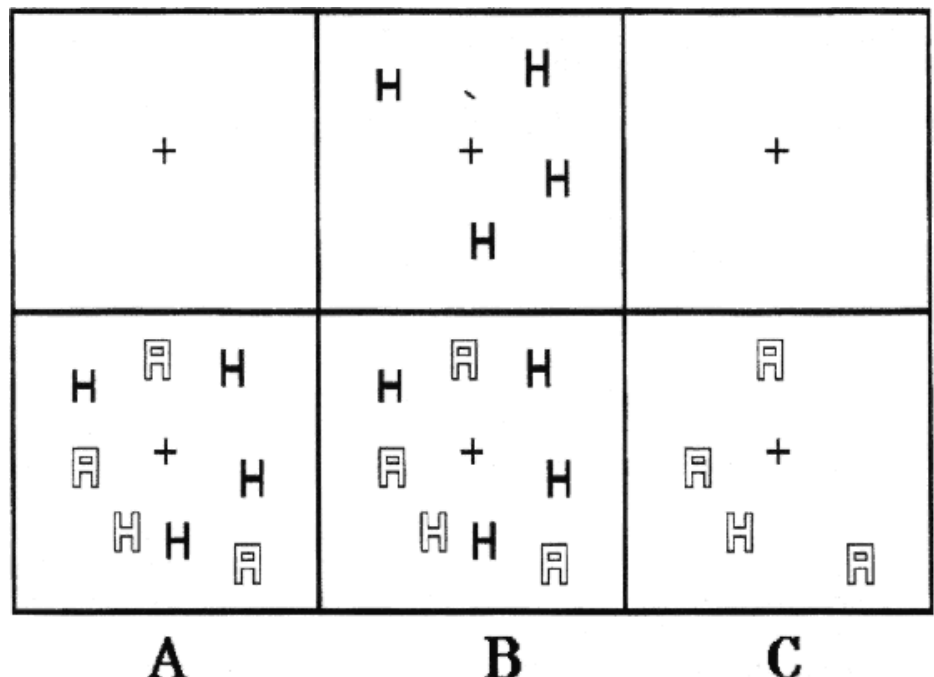

Figure 1. Examples of the three types of target-present search displays used by Watson and Humphreys (1997). Panel A depicts the all-element baseline condition, panel $B$ depicts the gap condition, and panel $C$ depicts the halfelem ent baseline condition. These displays were called the low-salience displays in Experiment 1. The solid letters appeared green, and the hollow letters appeared blue. Note that the displays are not drawn to scale.

target display (cf. Horowitz \& Wolfe, 1998; Klein \& Taylor, 1994).

However, although previous studies have shown that visual marking may improve the efficiency of visual search by reducing the size of the search set, it is important to realize that reducing the size of the search set is not the only way in which visual marking may come to improve the efficiency of visual search. For instance, suppose once again that the target was a blue $H$ and the distractors were green $H \mathrm{~s}$ and blue $A \mathrm{~s}$. Suppose further that the blue $A$ s (as opposed to the green $H$ s) were shown, $1,000 \mathrm{msec}$ before the appearance of the $H \mathrm{~s}$. In this situation, visual marking of the preceding blue $A$ s may improve the efficiency of visual search not only by reducing the size of the search set, but also by increasing the perceptual salience of the blue $H$ target; that is, successful visual marking of the old distractors may enable the target to appear as the sole blue $H$ among green $H$ s in the target display. This is important because the search for a salient target can usually be accomplished very efficiently, often in a single step, regardless of the number of distractors (Treisman, 1988, 1994; Treisman \& Gelade, 1980). Thus, increasing the perceptual salience of the target would likely be even more beneficial than simply reducing the size of the search set.

Unfortunately, previous studies have been unable to determine whether the perceptual salience of the target can be increased under gap-like conditions. This is because the target can be considered to be high in salience only when it appears unique within a primitive feature dimension, such as color, orientation, curvature, or motion (Wolfe, 1994, 1998), and previous studies have been designed in such a way that the target did not appear unique within one of these primitive feature dimensions (Theeuwes et al., 1998; Watson \& Humphreys, 1997). For instance, the blue $H$ target had the potential to be unique in form when it appeared with the blue $A$ distractors in the gap condition used by Watson and Humphreys (1997; see Figure 1); however, form has typically not functioned as a primitive feature in visual search (Wolfe, 1994, 1998). Consequently, for the purposes of visual search, the blue $H$ target could not have appeared salient in the context of the blue $A$ distractors in Watson and Humphreys study, even if the process of visual marking was capable of altering the perceptual context of the display (see also Theeuwes et al., 1998). Thus, although the evidence obtained from such studies has clearly shown that old distractors can be marked as irrelevant and then avoided during the course of an attentionally demanding search task, this evidence has no bearing on the question of whether this marking process can also be used to increase the perceptual salience of the target. ${ }^{2}$ Hence, the primary goal of the present study was to investigate the extent to which this marking process can be used to increase the efficiency of an attentionally demanding search task by increasing the perceptual salience of the target.

\section{EXPERIMENT 1}

In order to determine whether the relative salience of the target can be increased in the gap condition, two sets of three search displays were compared in Experiment 1. One set of displays was identical to the set used by Watson and Humphreys (1997) and is shown in Figure 1. This 


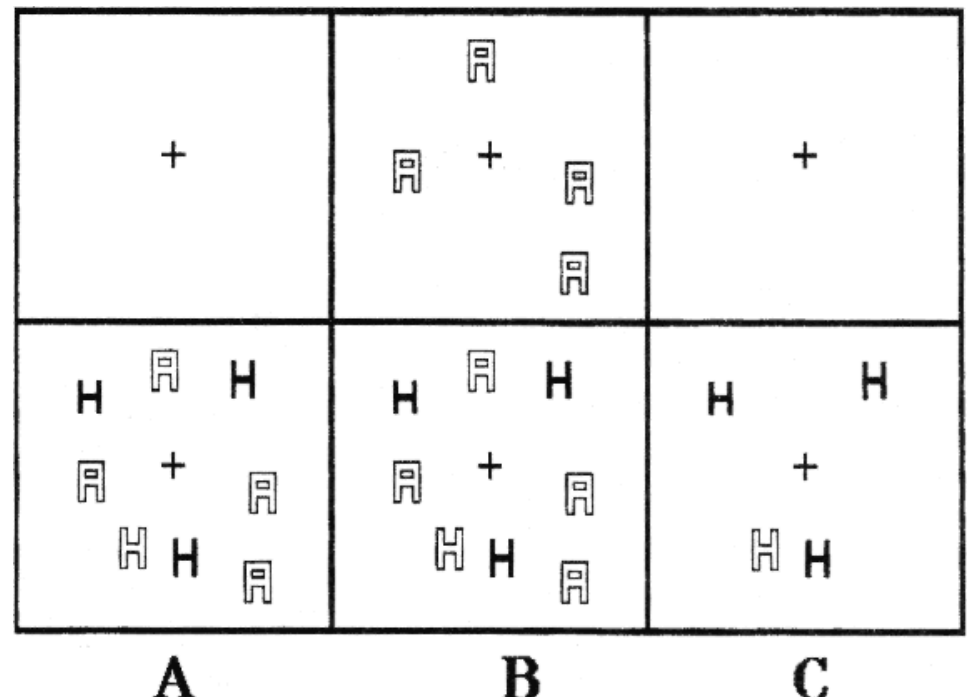

Figure 2. Modified versions of the three types of target-present search displays used by Watson and Humphreys (1997). Panel A depicts the all-element baseline condition, panel $B$ depicts the gap condition, and panel $C$ depicts the half-element baseline condition. These displays were called the high-salience displays in Experiment 1. The solid letters appeared green, and the hollow letters appeared blue. Note that the displays are not drawn to scale.

set of displays was called the low-salience set in the present experiment. The other set of displays, shown in Figure 2, involved the same stimuli as the low-salience set, except that the displays were rearranged so that the target would now be high in salience in the half-element baseline condition and would have the potential to be high in salience in the gap condition. Note that the target was relatively low in salience in both all-element baseline conditions shown in Figures 1 and 2; thus, the terms high salience and low salience will be used in reference to the all-element baseline condition simply to associate these conditions with their corresponding half-element and gap conditions.

For the purposes of this article, the target will be considered high in salience when it differs from a set of distractors on the basis of a primitive feature, such as color, orientation, or curvature (Wolfe, 1994, 1998). For instance, on target-present trials in the high-salience halfelement baseline condition, a blue $H$ target appeared among several green $H$ distractors. The blue $H$ target may be considered high in salience in this context, because blue is encoded as a different primitive feature than green (Wolfe, 1994, 1998). Under these conditions, existing evidence suggests that observers tend to divide their attention across the entire target display and then attempt to group the individual display elements into a single, global object so that the discrepant target element can be easily detected among the homogenous distractors (Nakayama \& Joseph, 1998; Treisman, 1994). Thus, the blue $H$ target should be detected very efficiently in the high-salience half-element baseline condition(D'Zmura, 1991; Nagy \& Sanchez, 1990; Treisman \& Gelade, 1980).

In contrast, the blue $H$ target may be considered low in salience when it appears with the blue $A$ distractors in the half-element baseline condition shown in Figure 1, because both stimuli were composed of similar primitive features. For instance, they both shared the same color, and they both shared many of the same line segments (both stimuli were created by erasing line segments from a block figure eight). Under these conditions, existing evidence suggests that observers tend to focus their attention more narrowly on individual display elements (Treisman, 1994), which may then help reduce the interference that can arise from distractors that are highly similar to the target (Duncan \& Humphreys, 1989; Theeuwes, 1990). Search is thus typically conducted in a more serial (and thus less efficient) manner when the target is relatively nonsalient. As a result, the target should be detected less efficiently in the low-salience half-element baseline condition than in the high-salience half-element baseline condition.

In the high-salience gap condition, a blue $H$ target also appeared (on target-present trials) at the same time as several green $H$ distractors, but this target display was added to a preexisting display of blue $A$ distractors. We expected one of two possible outcomes in this gap condition. On the one hand, successful visual marking of the old distractors may enable the new elements to be segregated perceptually from the old elements. Accordingly, the presence of 
the old distractors may not influence the perceived salience of the target. If so, the blue $H$ target should be detected more efficiently in the high-salience gap condition than it is in the all-element baseline condition, and it should be detected equally efficiently in both the high-salience gap condition and the high-salience half-element baseline condition.

On the other hand, however, the presence of the old distractors may decrease the salience of the target in the gap condition. For instance, the relatively low level perceptual processes underlying the computation of salience are based on hard-wired interactions in the brain (see, e.g., Braun, 1998; Desimone \& Duncan, 1995) that may not be capable of being modulated by visual marking. Indeed, the old distractors do remain visible and continue to be seen as blue even when they are successfully marked. Accordingly, the shared blueness of the old distractors and the target may cause the salience of the target to be diminished. This processing constraint will be referred to as the perceptual constraint hypothesis, to emphasize the role of bottom-up factors in the processing of visual salience. Alternatively, observers may also routinely search the set of new elements in the gap condition as though it were low in salience (by focusing attention on individual display elements, rather than by dividing attention across the display), perhaps because successful marking of the old elements is incompatible with a highsalience search strategy (see, e.g., Theeuwes, 1990, 1991, 1992). Indeed, existing evidence suggests that a lowsalience search strategy may sometimes be used in highsalience search tasks, resulting in search performance that is more characteristic of low-salience search tasks than it is of high-salience search tasks (Gibson \& Jiang, 1998). This processing constraint will be referred to as the attentional constraint hypothesis, to emphasize the role of topdown factors in the processing of visual salience. If either of these two hypotheses is correct, the blue $H$ target may be detected less efficiently in the high-salience gap condition than it is in the high-salience half-element baseline condition, although performance in this gap condition should still be better than performance in the corresponding all-element baseline condition (because the old distractors would presumably be marked and therefore excluded from the search).

Finally, in the low-salience gap condition, a blue $H$ target appeared (on target-present trials) at the same time as several blue $A$ distractors, but this target display was added to a preexisting display of green $H$ distractors. Regardless of the findings observed in the high-salience condition, we expected to replicate Watson and Humphreys's (1997) findings in the low-salience condition. That is, the blue $H$ target should be detected more efficiently in the lowsalience gap condition than it is in the all-element baseline condition, and it should be detected equally efficiently in both the low-salience gap condition and the low-salience half-element baseline condition. Such a replication would serve to show that the old distractors were, in fact, being excluded from the relevant search set, at least in the lowsalience gap condition.

\section{Method}

Subjects. A total of 38 University of Notre Dame undergraduates participated in this experiment for course credit. All the subjects reported normal color vision and normal or corrected-tonormal visual acuity.

Stimuli and Apparatus. The stimuli were $A$ s and $H$ s, each generated from a subset of a seven-segment figure eight. Each element subtended $0.92^{\circ} \times 0.46^{\circ}$ of visual angle on the computer screen, which was placed approximately $50 \mathrm{~cm}$ from the observer. Display elements were randomly positioned in the cells of an invisible $10 \times$ 10 matrix, which subtended $11.86^{\circ} \times 10.20^{\circ}$ of visual angle. The letters appeared either blue (IBM color 9) or green (IBM color 10) on a black background. Display size was 4,8 , and 16 in both the allelement baseline and the gap conditions, and it was 2, 4, and 8 in the half-element baseline condition. In the gap condition, half of the elements appeared in the preview display, and the other half of the elements (including the target, if present) appeared in the target display. The target was present on $50 \%$ of the trials in all the search conditions. In addition, half of the displays in each search condition were classified as high-salience displays, and the other half were classif ied as low-salience displays. In the half-element baseline condition, the high-salience displays contained a blue $H$ target and several green $H$ distractors (on target-present trials), whereas the low-salience displays contained a blue $H$ target and several blue $A$ distractors (on target-present trials). The high-salience and lowsalience displays shown in the gap condition were identical to those used in the half-element baseline condition, with the sole exception that several blue $A$ distractors preceded the high-salience target displays and several green $H$ distractors preceded the low-salience target displays. There were also subtle differences between the highsalience and the low-salience displays shown in the all-element baseline condition. For instance, the high-salience displays contained an equal number of $A \mathrm{~s}$ and $H \mathrm{~s}$ but an unequal number of green and blue elements on target-present trials, whereas the lowsalience displays contained an equal number of green and blue elements but an unequal number of $A$ s and $H$ s on target-present trials. The two types of displays were identical on target-absent trials (i.e., all the displays contained an equal number of $H \mathrm{~s}$ and $A$ s and an equal number of green and blue elements). Sample displays are shown in Figure 1 (low salience) and Figure 2 (high salience). ${ }^{3}$ All the stimuli were presented on a ZEOS color monitor equipped with a standard VGA video card. Response time and error rates were recorded by a ZEOS 486 microcomputer.

Procedure. Each trial of the experiment began with the appearance of a centrally located fixation cross that remained on the screen throughout the duration of the display sequence. The observers were instructed to keep their eyes focused on the cross throughout each trial, although eye movements were not monitored in this experiment. The half-element baseline, all-element baseline, and gap conditions were presented in separate blocks of trials, the order of which was counterbalanced across observers. In the gap condition, the preview display appeared $1,000 \mathrm{msec}$ after the appearance of the initial fixation cross and then remained on the screen for an additional $1,000 \mathrm{msec}$ before the target display was added. There was always an equal number of elements in the preview and target displays (two, four, or eight). The observers were told that the target would always appear in the second of the two displays, and they were instructed to withhold responding until the target display appeared. In the half-element baseline and all-element baseline conditions, the target display appeared 1,000 $\mathrm{msec}$ after the appearance of the initial fixation cross. In each condition, the target display remained visible until a response was provided or until $4 \mathrm{sec}$ 


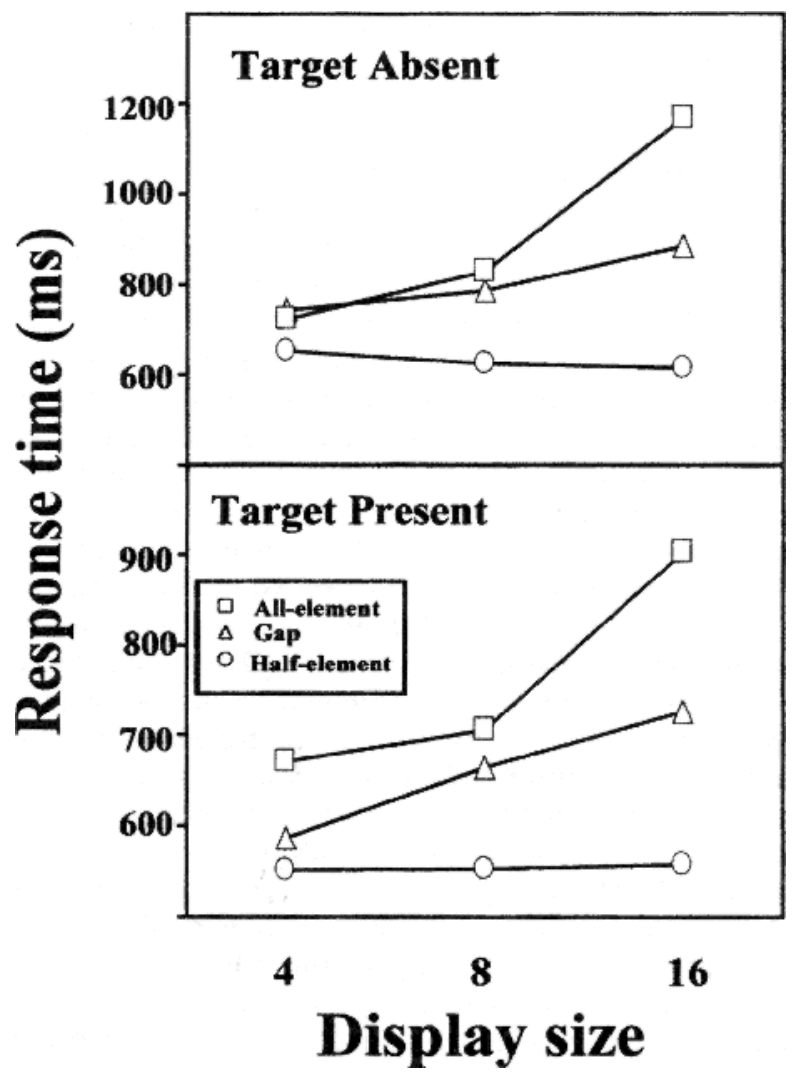

Figure 3. Mean correct response times in the high-salience display condition as a function of search type, target presence, and display size in Experiment 1 . The top panel depicts target-absent trials, and the bottom panel depicts target-present trials.

elapsed. The observers responded by pressing the right shift key to indicate that the target was present and the left shift key to indicate that the target was absent. The observers were instructed to respond as quickly as possible without sacrificing accuracy. Feedback was provided following errors to reinforce these instructions.

Each observer participated in a single session that lasted approximately $45 \mathrm{~min}$. Within these sessions, the observers completed three blocks of 252 trials (the first 12 of which in each block served as practice). Within each block, there was an equal number of highsalience and low-salience displays presented at each of the three display sizes. The order of trials within each block was randomly determined for each subject.

\section{Results and Discussion}

Mean correct response times (RTs) in both the highsalience and the low-salience conditions are shown in Figures 3 and 4, respectively, as a function of search type, target presence, and display size. Corresponding error rates and slopes are listed in Tables 1 and 2, respectively. Note that display size was doubled in all calculations of slope involving the half-element baseline condition so that this condition could be directly compared with the allelement baseline and gap conditions. As such, the slopes in the half-element baseline condition are half their actual value (see Watson \& Humphreys, 1997, for a more de- tailed discussion of this issue). Note also that, for the sake of discussion, the present analyses focused mainly on mean correct RTs. This is because the pattern of errors always mirrored the pattern of RTs in the present series of experiments, and thus there was no evidence for a speed/ accuracy tradeoff.

Half-element baseline condition. As was expected, visual search was performed more efficiently in the high-salience condition than in the low-salience condition, on both target-present and target-absent trials. As can be seen in Table 2, the average slope in the highsalience condition was $0.52 \mathrm{msec} /$ item when the target was present and $-2.86 \mathrm{msec} /$ item when the target was absent, whereas the average slope in the low-salience condition was $13.48 \mathrm{msec} / \mathrm{item}$ when the target was present and $20.24 \mathrm{msec} /$ item when the target was absent. This difference in search efficiency was also supported by a significant salience type $\times$ display size interaction in both the target-present condition $\left[F(2,72)=51.93, M S_{\mathrm{e}}=\right.$ $2,321.84, p<.0001]$ and the target-absent condition $\left[F(2,72)=74.50, M S_{\mathrm{e}}=5,161.66, p<.0001\right]$.

Half-element baseline condition versus gap condition. If the relative salience of the target is unaffected by the presence of the old distractors, visual search performance should be equally efficient in both the gap and the half-element baseline conditions. However, the present results suggested otherwise. As can be seen in Table 2, the average slope in the high-salience gap condition was $11.04 \mathrm{msec} /$ item when the target was present and $11.86 \mathrm{msec} /$ item when the target was absent. When compared with the corresponding half-element baseline condition, visual search was consistently observed to be less efficient in the high-salience gap condition than in the high-salience half-element baseline condition, regardless of whether the target was present or absent $[F(2,72)=$ $30.51, M S_{\mathrm{e}}=2,781.01, p<.0001$, and $F(2,72)=22.29$, $M S_{\mathrm{e}}=6,943.80, p<.0001$, respectively]. Note, however, that the apparent influence of the old distractors on search performance was confined to the high-salience gap condition. As can be seen in Table 2, the average slope in the low-salience gap condition was $12.23 \mathrm{msec} / \mathrm{item}$ when the target was present and $16.48 \mathrm{msec} /$ item when the target was absent. As was expected, when compared with the corresponding half-element baseline condition, planned comparisons showed that visual search was performed just as efficiently in the low-salience gap condition as in the low-salience half-element baseline condition when the target was present $(F<1)$, and visual search was actually performed more efficiently in the low-salience gap condition than in the low-salience half-element baseline condition when the target was absent $\left[F(2,72)=3.09, M S_{\mathrm{e}}=\right.$ $8,968.27, p=.05]$. This latter finding appears to be due solely to the occurrence of unusually long RTs when four elements appeared in the gap condition (i.e., two in the preview display and two in the target display), a result that was also observed in some of Watson and Humphreys's (1997) experiments (see, especially, Experiment 1). As such, the present results obtained in the low-salience con- 


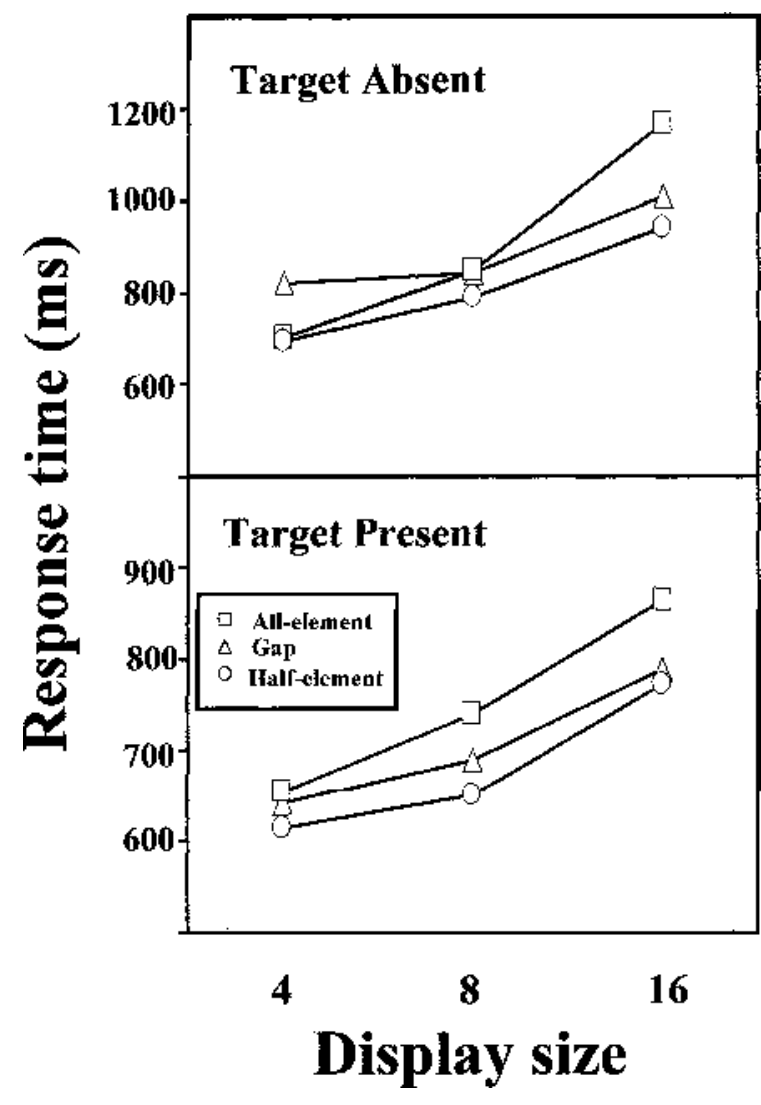

Figure 4. Mean correct response times in the low-salience display conditions as a function of search type, target presence, and display size in Experiment 1. The top panel depicts target-absent trials, and the bottom panel depicts target-present trials.

dition replicate the main findings reported by Watson and Humphreys. The different pattern of results observed across the high-salience and low-salience conditions was also supported by a significant search type $X$ salience type $X$ display size interaction in both the target-present condition $\left[F(2,72)=19.80, M S_{\mathrm{e}}=2,527.55, p<.0001\right]$ and the target-absent condition $\left[F(2,72)=23.46, M S_{\mathrm{e}}=\right.$ $5,736.65, p<.0001]$.

All-element baseline condition versus gap condition. Although visual search was performed less efficiently in the high-salience gap condition than in the high-salience half-element baseline condition, planned comparisons showed that visual search was nevertheless consistently performed more efficiently in the highsalience gap condition than in the corresponding allelement baseline condition, regardless of whether the target was present or absent $\left[F(2,72)=19.28, M S_{\mathrm{e}}=\right.$ $4,687.80, p<.0001$, and $F(2,72)=51.74, M S_{\mathrm{e}}=9,510.06$, $p<.0001$, respectively; the average slope in this allelement baseline condition was $20.09 \mathrm{msec} / \mathrm{item}$ when the target was present and $37.96 \mathrm{msec} /$ item when the target was absent]. Thus, the temporal separation of the preview and the target displays in the high-salience gap con- dition did provide some benefit to finding the target. In addition, consistent with Watson and Humphreys's (1997) findings, planned comparisons also showed that visual search was performed more efficiently in the low-salience gap condition than in the corresponding all-element baseline condition, regardless of whether the target was present or absent $\left[F(2,72)=4.80, M S_{\mathrm{e}}=3,929.93, p<\right.$ .02 , and $F(2,72)=35.23, M S_{\mathrm{e}}=10,099.21, p<.0001$, respectively; the average slope in this all-element baseline condition was $17.14 \mathrm{msec} /$ item when the target was present and $38.68 \mathrm{msec} / \mathrm{item}$ when the target was absent].

In summary, the present study compared the effectiveness of visual marking across two visual search contexts. The low-salience gap condition was identical to the gap condition used in many of Watson and Humphreys's (1997) experiments. Consistent with Watson and Humphreys's previous findings, the present results suggested that the old distractors were successfully marked: Visual search was performed just as efficiently in the low-salience gap condition as in the low-salience half-element baseline condition. In addition, visual search was also performed more efficiently in the low-salience gap condition than in the corresponding all-element baseline condition. These findings therefore suggest that observers successfully avoided searching the locations occupied by the old distractors in the low-salience gap condition.

Of more critical importance to the present study were the results obtained in the high-salience gap condition in which the target had the potential to appear as a color singleton. Visual search had the potential to be improved even more dramatically in this condition than in the lowsalience gap condition, assuming that the presence of the old distractors did not interfere with the high-salience search task. Contrary to this hypothesis, however, the results suggested that the presence of the old distractors did somehow influence the salience of the target, in that visual search was performed less efficiently in the high-salience gap condition than in the high-salience half-element baseline condition. However, although these findings suggest that the presence of the old, blue distractors diminished the salience of the target, search nevertheless appeared to be confined to only half of the elements in the high-salience gap condition. This conclusion follows from the finding that visual search was performed almost twice as fast in the high-salience gap condition as in the corresponding all-element baseline condition.

Together, these findings suggest that visual marking may be limited in its ability to increase the efficiency of an attentionally demanding search task. In particular, the present findings suggest that visual marking can improve the efficiency of visual search by reducing the size of the search set; however, visual marking may be limited in its ability to increase the perceptual salience of the target. As was mentioned in the introduction to Experiment 1, the pattern of results obtained in the high-salience condition is consistent with a variety of different processing constraints. Thus, additional evidence will be required to understand exactly how the old distractors affect search ef- 
Table 1

Mean Error Rates (\%) in Experiment 1 Listed as a Function of Search Type, Salience Type, Target Presence, and Display Size

\begin{tabular}{ccccccc}
\hline & & \multicolumn{2}{c}{ Target Absent } & & \multicolumn{2}{c}{ Target Present } \\
\cline { 3 - 4 } \cline { 6 - 7 } Condition & Display Size & High Salience & Low Salience & & High Salience & Low Salience \\
\hline Gap & 4 & 2.62 & 3.95 & & 4.87 & 2.24 \\
& 8 & 2.89 & 2.10 & & 5.00 & 3.29 \\
All-element & 16 & 2.10 & 2.63 & & 15.66 & 8.29 \\
& 4 & 2.76 & 1.44 & & 4.21 & 3.16 \\
& 8 & 1.97 & 1.58 & & 3.82 & 5.40 \\
Half-element & 16 & 1.84 & 3.29 & & 12.24 & 10.00 \\
& 4 & 2.10 & 3.42 & & 1.98 & 4.22 \\
& 8 & 1.84 & 2.37 & & 1.71 & 4.60 \\
& 16 & 2.10 & 4.60 & & 1.84 & 11.98 \\
\hline
\end{tabular}

ficiency in the high-salience gap condition. We will begin, however, by considering a potential confound that was present between the high-salience and the low-salience gap conditions in Experiment 1.

\section{EXPERIMENT 2}

The first experiment showed that visual search was affected by old distractors more in the high-salience gap condition than in the low-salience gap condition. Note, however, that the target was always more similar to the old distractors in the high-salience gap condition than in the low-salience gap condition used in Experiment 1 (see Figures 1 and 2). Hence, the high-salience targets may have been harder to detect than the low-salience targets in the gap condition (relative to the corresponding halfelement baseline conditions) because the effectiveness of visual marking may depend on the relative similarity between the target and the old distractors (cf. Theeuwes et al., 1998).

Experiment 2 was conducted to examine whether the presence of the old distractors would continue to influence search performance in the high-salience gap condition under conditions in which the target was identical to the old distractors on one feature dimension and highly distinguishable from the old distractors on the other feature dimension (as it was in the low-salience gap condition used in Experiment 1). This goal was accomplished by using colors and forms that were both highly distinguishable. In particular, the target was a blue $X$ and the distractors were blue $O$ s and green $X$ s in Experiment 2. Thus, like color, the two forms could be distinguished on the basis of a primitive feature (such as orientation or curvature) in this experiment. As in the previous experiment, two sets of three visual search displays were created from these stimuli (the all-element baseline condition, the gap condition, and the half-element baseline condition). However, unlike in the first experiment, the blue $X$ target was expected to be highly salient in both sets. Thus, if the perceptual similarity between the old elements and the target can diminish the relative salience of the target, visual search should be performed less efficiently in both gap conditions in the present experiment than in their respective half-element baseline conditions.

\section{Method}

Subjects. A total of 18 University of Notre Dame undergraduates participated in this experiment for course credit. All the subjects reported normal color vision and normal or corrected-tonormal visual acuity.

Stimuli and Apparatus. The stimuli were $X$ s and $O$ s that subtended approximately $0.92^{\circ} \times 0.46^{\circ}$ of visual angle on the computer screen, which was placed approximately $50 \mathrm{~cm}$ from the observer. The letters appeared either blue or green on a black background (as in Experiment 1). For the sake of clarity, the two sets of displays were called the color-salience condition and the form-salience condition in the present experiment (although it is should be noted that a form would appear salient in the present experiment because of differences in orientation and/or curvature). In the half-element baseline condition, the color-salience displays contained a blue $X$ target and several green $X$ distractors (on target-present trials), whereas the form-salience displays contained a blue $X$ target and several blue $O$ distractors (on target-present trials). The colorsalience and form-salience displays shown in the gap condition were identical to those used in the half-element baseline condition, with the sole exception that several blue $O$ distractors preceded the color-salience target displays and several green $X$ distractors preceded the form-salience target displays. As in the previous two experiments, there were subtle differences between the color-salience

Table 2

Slopes of the Display-Size Functions in Experiment 1 Listed as a Function of Search Type, Salience Type, and Target Presence (With $R^{2}$ )

\begin{tabular}{|c|c|c|c|c|c|c|c|c|}
\hline \multirow[b]{3}{*}{ Display size } & \multicolumn{4}{|c|}{ Target Absent } & \multicolumn{4}{|c|}{ Target Present } \\
\hline & \multicolumn{2}{|c|}{ High Salience } & \multicolumn{2}{|c|}{ Low Salience } & \multicolumn{2}{|c|}{ High Salience } & \multicolumn{2}{|c|}{ Low Salience } \\
\hline & Average & $R^{2}$ & Average & $R^{2}$ & Average & $R^{2}$ & Average & $R^{2}$ \\
\hline Gap & 11.86 & 1.00 & 16.48 & 0.88 & 11.04 & 0.90 & 12.23 & 0.99 \\
\hline All-element & 37.96 & 0.99 & 38.68 & 1.00 & 20.09 & 0.98 & 17.14 & 0.98 \\
\hline Half-element & -2.86 & 0.94 & 20.24 & 1.00 & 0.52 & 0.98 & 13.48 & 0.97 \\
\hline
\end{tabular}




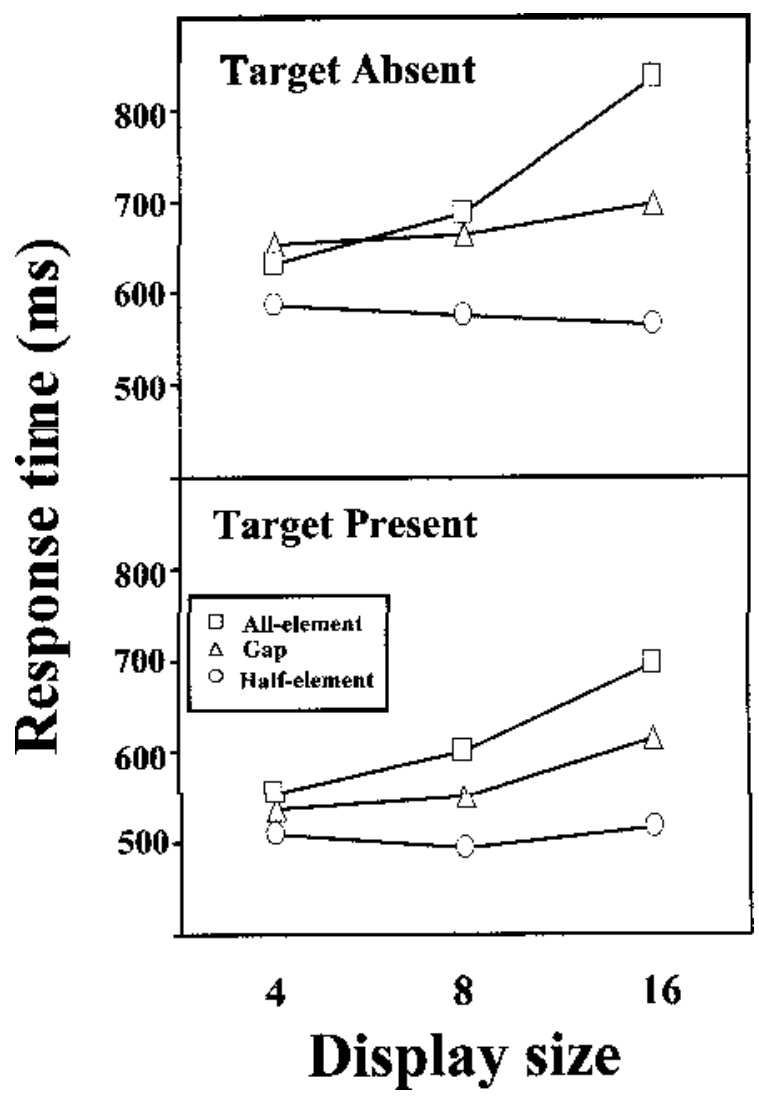

Figure 5. Mean correct response times in the color-salience display condition as a function of search type, target presence, and display size in Experiment 2. The top panel depicts target-absent trials, and the bottom panel depicts target-present trials.

and form-salience displays shown in the all-element baseline condition. For instance, the color-salience displays contained an equal number of $X \mathrm{~s}$ and $O \mathrm{~s}$, but an unequal number of blue and green elements on target-present trials, whereas the form-salience displays contained a equal number of blue and green elements, but an unequal number of $X \mathrm{~s}$ and $O \mathrm{~s}$ on target-present trials. The two types of displays were identical on target-absent trials (i.e., all the displays contained an equal number of $X \mathrm{~s}$ and $O \mathrm{~s}$ and an equal number of blue and green elements). All other aspects of this experiment were identical to those in Experiment 1.

Procedure. The procedure was identical to that in Experiment 1.

\section{Results and Discussion}

Mean correct RTs in the color-salience and formsalience conditions are shown in Figures 5 and 6, respectively, as a function of search type, target presence, and display size. Corresponding error rates and slopes are listed in Tables 3 and 4, respectively.

Half-element baseline condition. As was expected, visual search was found to be highly efficient in both the form and the color half-element baseline conditions when the target was present. As can be seen in Table 4, the slope in the color-salience half-element baseline condition was $0.95 \mathrm{msec} / \mathrm{item}$ on target-present trials, and the slope in the form-salience half-element baseline condition was
$1.84 \mathrm{msec} /$ item on target-present trials. The only significant effect observed in this analysis was that RTs were overall faster in the color-salience half-element baseline condition than in the form-salience half-element baseline condition $\left[F(1,17)=22.06, M S_{\mathrm{e}}=1,050.51, p<.0005\right]$ for the main effect of salience type. Similarly, visual search was also found to be highly efficient on target-absent trials as well: The slope in the color half-element baseline condition was $-1.61 \mathrm{msec} /$ item on target-absent trials, whereas the slope in the form half-element baseline condition was $0.21 \mathrm{msec} /$ item on target-absent trials. As on target-present trials, RTs were also faster in the colorsalience half-element baseline condition than in the formsalience half-element baseline condition $[F(1,17)=6.75$, $\left.M S_{\mathrm{e}}=3,179.61, p<.02\right]$ for the main effect of salience type.

Half-element baseline condition versus gap condition. A more critical goal of Experiment 2 was to determine whether search efficiency would continue to be affected by the old distractors in both the color-salience and form-salience gap conditions even though the target was now highly distinguishable from the old distractors along at least one featural dimension (i.e., either color or form). As can be seen in Table 4, the slope in the color-

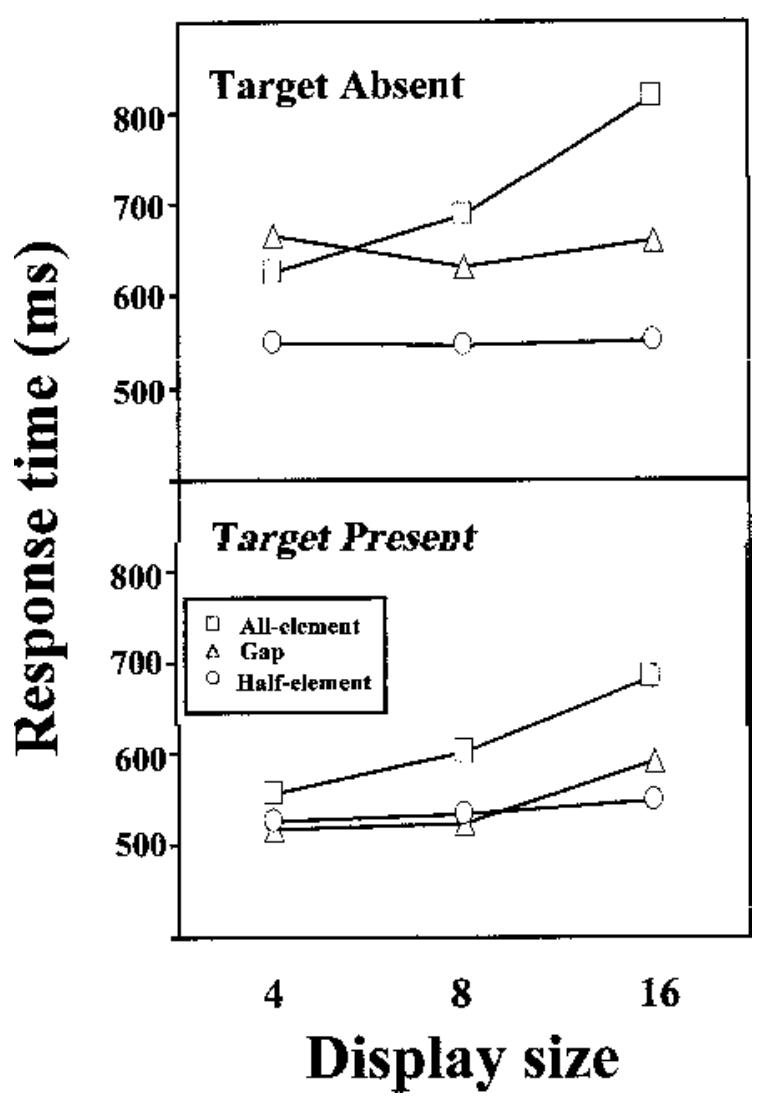

Figure 6. Mean correct response times in the form-salience display condition as a function of search type, target presence, and display size in Experiment 2 . The top panel depicts target-absent trials, and the bottom panel depicts target-present trials. 
Table 3

Mean Error Rates (\%) in Experiment 2 Listed as a Function of Search Type, Salience Type, Target Presence, and Display Size

\begin{tabular}{|c|c|c|c|c|c|}
\hline \multirow[b]{2}{*}{ Condition } & \multirow[b]{2}{*}{ Display Size } & \multicolumn{2}{|c|}{ Target Absent } & \multicolumn{2}{|c|}{ Target Present } \\
\hline & & Color Salience & Form Salience & Color Salience & Form Salience \\
\hline \multirow[t]{3}{*}{ Gap } & 4 & 2.50 & 1.11 & 1.94 & 1.11 \\
\hline & 8 & 1.39 & 1.67 & 2.78 & 0.56 \\
\hline & 16 & 1.94 & 1.39 & 6.39 & 2.50 \\
\hline \multirow[t]{3}{*}{ All-element } & 4 & 1.11 & 1.11 & 1.11 & 3.06 \\
\hline & 8 & 1.39 & 1.11 & 2.50 & 4.72 \\
\hline & 16 & 0.83 & 2.22 & 6.94 & 7.50 \\
\hline \multirow[t]{3}{*}{ Half-element } & 4 & 3.06 & 0.28 & 1.67 & 1.39 \\
\hline & 8 & 1.11 & 1.67 & 4.17 & 1.39 \\
\hline & 16 & 0.83 & 1.67 & 2.50 & 2.22 \\
\hline
\end{tabular}

salience gap condition was $6.71 \mathrm{msec} /$ item on targetpresent trials, and the slope in the form-salience gap condition was $6.41 \mathrm{msec} / \mathrm{item}$ on target-present trials. In accordance with Experiment 1, the results obtained on target-present trials indicated that search efficiency continued to be impaired in both the color-salience and the form-salience gap conditions, relative to the corresponding color-salience and form-salience half-element baseline conditions $\left[F(2,34)=7.71, M S_{\mathrm{e}}=2,349.93, p<\right.$ $.002]$, for the search type $\times$ display size interaction. The search type $X$ salience type $X$ display size interaction did not approach significance on target-present trials in Experiment $2(F<1)$. These results therefore provide strong evidence that the shared perceptual features of the old distractors can diminish the salience of the target, even when the target is highly distinguishablefrom the old distractors along at least one feature dimension. However, it should also be noted that the results obtained on targetabsent trials were not quite so clear-cut. As can be seen in Table 4, the slope in the color-salience gap condition was $3.80 \mathrm{msec} /$ item on target-absent trials, and the slope in the form-salience gap condition was $0.21 \mathrm{msec} / \mathrm{item}$ on target-absent trials. When compared with their corresponding half-element baseline conditions, the results showed that visual search was again performed less efficiently in the color-salience gap condition than in the color-salience half-element baseline condition $[F(2,34)=$ $\left.3.13, M S_{\mathrm{e}}=3,127.73, p=.056\right]$; however, visual search was performed equally efficiently in both the form-salience gap condition and the form-salience half-element baseline condition $\left[F(2,34)=1.19, M S_{\mathrm{e}}=2,035.61, p>.30\right]$. Such findings again appear to be due to unusually long RTs in the smallest display-size condition. Altogether, the differential pattern of results observed across the colorsalience and form-salience conditions produced a significant search type $\times$ salience type $X$ display size interaction $\left[F(2,34)=3.61, M S_{\mathrm{e}}=1,710.32, p<.05\right]$.

All-element baseline condition versus gap condition. Consistent with the previous experiment, the results obtained in Experiment 2 again showed a benefit in search efficiency in both gap conditions, relative to the corresponding all-element baseline conditions. As can be seen in Table 4, the slope in the color-salience all-element baseline condition was $11.77 \mathrm{msec} /$ item on target-present trials, and the slope in the form-salience all-element baseline condition was $10.60 \mathrm{msec} /$ item on target-present trials. In the target-present condition, there was a significant search type $\times$ display size interaction $[F(2,34)=$ $\left.10.40, M S_{\mathrm{e}}=1,530.23, p<.0005\right]$ and a nonsignificant search type $\times$ salience type $\times$ display size interaction $(F<1)$. Likewise, a similar pattern was observed in the target-absent condition. As can be seen in Table 4, the slope in the color-salience all-element baseline condition was $16.89 \mathrm{msec} /$ item on target-absent trials, and the slope in the form-salience all-element baseline condition was $16.03 \mathrm{msec} /$ item on target-absent trials. Thus, in the target-absent condition, there was a significant search type $\times$ display size interaction $\left[F(2,34)=19.22, M S_{\mathrm{e}}=\right.$ $7,341.20, p<.0001]$ and a nonsignificant search type $\times$ salience type $\times$ display size interaction $[F(2,34)=1.34$, $\left.M S_{\mathrm{e}}=2,406.75, p>.25\right]$.

In summary, Experiment 2 was conducted to ensure that the different pattern of results observed across the two gap conditions in Experiment 1 was due to differences in the target's salience, rather than to differences in perceptual similarity between the target and the old distractors.

Table 4

Slopes of the Display-Size Functions in Experiment 2 Listed as a Function of Search Type, Salience Type, Target Presence (With $R^{2}$ )

\begin{tabular}{|c|c|c|c|c|c|c|c|c|}
\hline \multirow[b]{3}{*}{ Display Size } & \multicolumn{4}{|c|}{ Target Absent } & \multicolumn{4}{|c|}{ Target Present } \\
\hline & \multicolumn{2}{|c|}{ Color Salience } & \multicolumn{2}{|c|}{ Form Salience } & \multicolumn{2}{|c|}{ Color Salience } & \multicolumn{2}{|c|}{ Form Salience } \\
\hline & Average & $R^{2}$ & Average & $R^{2}$ & Average & $R^{2}$ & Average & $R^{2}$ \\
\hline Gap & 3.80 & 0.99 & 0.21 & 0.01 & 6.71 & 0.98 & 6.41 & 0.94 \\
\hline All-element & 16.89 & 1.00 & 16.03 & 1.00 & 11.77 & 1.00 & 10.60 & 1.00 \\
\hline Half-element & -1.60 & 0.91 & 0.21 & 0.31 & 0.95 & 0.25 & 1.84 & 1.00 \\
\hline
\end{tabular}


The results of Experiment 2 showed that the old distractors generally continued to impair the detection of a highly salient target even when the target shared only one feature with the old distractors (as it did in the low-salience gap condition used in Experiment 1). Thus, decreasing the similarity between the target and the old distractors does not appear to eliminate the difference in search efficiency observed between the high-salience gap condition and the half-element baseline condition.

Conversely, it is also worth pointing out that other studies have found that increasing the similarity between the target and the old distractors does not appear to decrease the efficiency of visual search when the target happens to be low in salience in the gap condition. For instance, Theeuwes et al. (1998) conducted a gap experiment in which both the preview and the target displays were highly similar, thus eliminating the possibility that featural differences between the old and the new displays might facilitate the benefit observed in the gap condition. This was accomplished by using a relatively demanding letter search task in which all the display elements (in both the preview and the target displays) appeared white. Moreover, both sets of distractors consisted of a heterogeneous display of letters, which is known to produce relatively inefficient visual search (Wolfe, 1998). Most important, the results showed that the observers appeared to limit their search for the target solely to the set of new elements. These findings therefore strongly suggest that visual search can be performed just as efficiently in the gap condition as in the half-element baseline condition when the target is low in salience, regardless of how similar the target is to the old distractors.

\section{EXPERIMENT 3}

The results of the first two experiments have suggested that the detection of a potentially salient target is affected more by the presence of old distractors than is the detection of a nonsalient target. However, at the present point in time, this evidence is consistent with at least two different kinds of processing constraints: a bottom-up, perceptually based constraint and a top-down, attentionally based constraint. Experiment 3 was therefore designed to further investigate the nature of the underlying processes.

One fundamental empirical issue that can serve to differentiate between the perceptual constraint hypothesis and the attentional constraint hypothesis concerns the question of whether search efficiency in the high-salience gap condition is affected primarily by the number of old elements or by the number of new elements. This issue could not be adequately addressed in the previous two experiments because the number of elements appearing in the preview display was confounded with the number of elements appearing in the target display. In the present experiment, however, the number of old elements (two, four, or eight) was varied independent of the number of new elements (two, four, or eight), which allowed us to in- vestigate the primary determinants of performance in the high-salience gap condition. In addition, the high-salience half-element condition was also included in the present experiment to serve as a baseline. With this in mind, let us now consider some specific predictions associated with the perceptual constraint hypothesis and the attentional constraint hypothesis.

According to one version of the perceptual constraint hypothesis, visual salience is thought to be based on inhibitory interactions that arise between neurons with similarly tuned, but nonoverlapping, receptive fields at a variety of visual areas in the brain (Braun, 1998; Desimone \& Duncan, 1995). Consider a neuron in area V4 of the primate visual system that responds optimally when a blue stimulus is presented within its receptive field. Although the firing rate of this neuron will generally not be decreased by the presence of green stimuli that are displayed outside the classic receptive field of this neuron (indeed, the firing rate of the blue neuron may even be enhanced), the firing rate of this neuron will tend to be decreased by the presence of other blue stimuli (Desimone, Schein, Moran, \& Ungerleider, 1985). Similar observations have been made also with respect to the neural representation of orientation in visual area V1 (Knierim \& Van Essen, 1992). Thus, at the neural level, the relative salience of the display elements may be understood in terms of the relative firing rates of neurons. Generally speaking, firing rate will be high when the appearance of a basic feature is unique and will typically decrease as similar elements are added to the visual field.

Notice that such a mechanism would signal that the blue $H$ target was salient in the high-salience gap condition (shown in Figure 2) only if the inhibitory interactions arising from the old, blue distractors were successfully modulated by visual marking. Thus, one reason the target may be harder to detect in the presence of old distractors than in their absence is because visual marking may not be capable of completely eliminating the bottom-up, inhibitory influence of the old distractors, which is likely to grow stronger (and therefore harder to suppress) as the number of old distractors increases.

Therefore, according to this perceptual constraint hypothesis, the salience of the target should decrease as the number of old elements is increased from two to eight. If search efficiency is measured as the time taken to search through increasing numbers of new elements, there should be two important consequences associated with increasing the number of old elements. First, search through the set of new elements should become progressively less efficient as the number of old elements is increased. In other words, target detection may be relatively unaffected by the number of new elements when there are only two old elements present in the display (much like the half-element baseline condition), whereas target detection may be strongly affected by the number of new elements when there are eight old elements present in the display. Second, search through a given set of new elements should also become progressively slower as the num- 


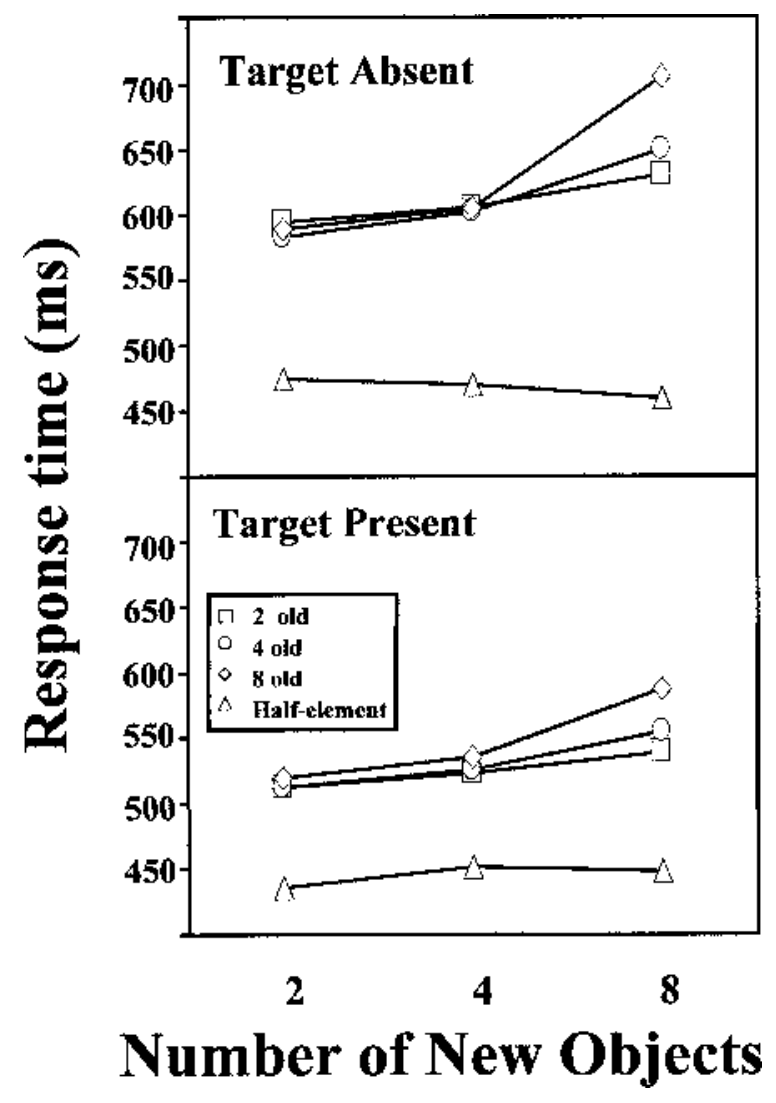

Figure 7. Mean correct response times in each of the targetpresent and target-absent conditions as a function of the number of new elements and the number of old elements in Experiment 3. The top panel depicts target-absent trials, and the bottom panel depicts target-present trials.

ber of old elements is increased. For instance, consider the condition in which only two new elements appear. In this condition, target detection should be slower when eight old elements are present in the display than when only two old elements are present in the display.

In contrast, according to the attentional constraint hypothesis, the highly efficient form of search that characterizes performance in the high-salience half-element baseline condition requires attention to be divided across the entire display; however, this search strategy may not be practical in the gap condition, where there are other (old) distractors that need to be ignored. For instance, there is evidence that certain forms of goal-directed selection are sacrificed during visual search when attention is divided, as compared with when it is focused (Theeuwes, 1990, 1991, 1992). In the present context, dividing attention across the display may have made it difficult to segregate the perceptual qualities of the old elements from the perceptual qualities of the new elements. Thus, successful visual marking of the old distractors may require a relatively narrow attentional focus, regardless of whether the target is high or low in salience. If this hypothesis is correct, attention should be allocated to indi- vidual display elements within the target display in a more serial (and thus less efficient) fashion; consequently, search should be affected only by the number of new elements in the present experiment. That is, search through the set of new elements should be equally inefficient regardless of the number of old elements.

\section{Method}

Subjects. A total of 21 University of Notre Dame undergraduates participated in this experiment for course credit. All the subjects reported normal color vision and normal or corrected-to-normal visual acuity.

Stimuli and Apparatus. The stimuli were the $H$ s and $A$ s used in Experiment 1. However, unlike in Experiment 1, only the highsalience gap and the high-salience half-element baseline conditions were included in the present experiment. Consequently, the observers always searched for a blue $H$ target among green $H$ distractors in the half-element baseline condition. Likewise, observers also searched for a blue $H$ target among green $H$ distractors in the gap condition; however, in addition, several blue $A$ distractors always appeared 1,000 msec earlier in the preview display. As in Experiment 1 , display size varied from 2 to 8 in the half-element baseline condition and from 4 to 16 in the gap condition. As was mentioned above, in the gap condition, the number of old elements $(2,4$, or 8$)$ was completely crossed with the number of new elements $(2,4$, or 8$)$.

Procedure. The procedure was similar to that in Experiment 1, except that only the high-salience half-element baseline and gap conditions were shown. As in Experiment 1, the observers participated in 252 trials in the half-element baseline condition (the first 12 of which were practice). In the gap condition, however, the observers participated in 372 trials (the first 12 of which again served as practice). There were an equal number of gap trials in each of the 2 target present $\times 3$ old object $\times 3$ new object conditions, and the order of trials was randomly determined by the computer for each observer.

\section{Results and Discussion}

Mean correct RTs in the gap and half-element baseline conditions are shown in Figure 7 as a function of target presence, the number of old elements (two, four, or eight), and the number of new elements (two, four, or eight). Corresponding error rates and slopes are listed in Tables 5 and 6 , respectively. Note that, in this experiment, unlike in the previous two, the slopes were calculated on the basis of the number of new elements that appeared in the display, regardless of how many old elements appeared in the gap condition. This is because the total number of display elements that appeared in the gap condition on any given trial was no longer double the total number of display elements that appeared in the half-element baseline condition.

The present experiment was designed to investigate the nature of the processes that underlie performance in the high-salience gap condition. In accordance with the perceptual constraint hypothesis, the present results showed that search through increasingly larger numbers of new elements did become progressively less efficient as the number of old elements appearing in the preview display increased from two to eight. More specifically, when the target was present, the slopes of the display-size functions for new elements increased from 4.24 msec/item to $11.48 \mathrm{msec} / \mathrm{item}$ as the number of old objects increased from two to eight, respectively. Likewise, when the target was absent, these slopes increased from $6.03 \mathrm{msec} /$ item 
Table 5

Mean Error Rates (\%) in Experiment 3 Listed as a

Function of Search Type, Target Presence, the Number of New Elements, and the Number of Old Elements

\begin{tabular}{|c|c|c|c|c|c|}
\hline \multirow[b]{2}{*}{ Condition } & \multirow[b]{2}{*}{ New Elements } & \multicolumn{4}{|c|}{ Old Elements } \\
\hline & & 0 & 2 & 4 & 8 \\
\hline \multicolumn{6}{|c|}{ Target Absent } \\
\hline \multirow{3}{*}{ Gap } & 2 & & 1.90 & 1.67 & 2.62 \\
\hline & 4 & & 0.95 & 2.38 & 0.48 \\
\hline & 8 & & 2.86 & 2.14 & 2.62 \\
\hline \multicolumn{6}{|c|}{ Target Present } \\
\hline & 2 & & 2.38 & 2.86 & 2.38 \\
\hline & 4 & & 1.90 & 2.86 & 4.76 \\
\hline & 8 & & 2.38 & 5.00 & 9.05 \\
\hline \multicolumn{6}{|c|}{ Target Absent } \\
\hline \multirow[t]{7}{*}{ Half-element } & & 1.43 & & & \\
\hline & & 1.43 & & & \\
\hline & & 0.95 & & & \\
\hline & \multicolumn{5}{|c|}{ Target Present } \\
\hline & & 1.19 & & & \\
\hline & & 4.05 & & & \\
\hline & & 1.90 & & & \\
\hline
\end{tabular}

to $20.13 \mathrm{msec} /$ item as the number of old objects increased from two to eight, respectively. This interpretation is supported by a significant old element $\times$ new element interaction $\left[F(4,80)=5.53, M S_{\mathrm{e}}=2,224.42, p<.0001\right]$; no other interactions were found to be significant (all $p \mathrm{~s}>$ .10). In addition, for the sake of comparison, it is also interesting to note that the observers consistently searched more slowly and less efficiently in the gap condition than they did in the half-element baseline condition-although the difference in search efficiency did not quite reach significance when the target was present and only two old distractors were shown $\left[F(2,40)=2.08, M S_{\mathrm{e}}=\right.$ $570.05, p>.10$ ]; however, search was consistently slower and less efficient in all the other conditions $(p<.05$ or less).

Note, however, that although certain aspects of the present findings were consistent with the perceptual con- straint hypothesis, other aspects of the present findings were not consistent with this hypothesis. In particular, search through a given set of new elements did not always become slower as the number of old elements increased. This inconsistency was especially clear when two new elements appeared in the target display. According to the perceptual constraint hypothesis, RT to detect the target should have been significantly slower when eight old elements were present in the display than when only two or four old elements were already present in the display. This is because increasing numbers of old elements should systematically decrease the salience of the target. However, the results of the present experiment clearly violated this prediction (see Figure 7). RTs were essentially identical across these three old-element conditions both when two new elements appeared in the target display and when four new elements appeared in the target display (both $F_{\mathrm{S}}<1$ ). Thus, this aspect of the present results is inconsistent with the perceptual constraint hypothesis and is also likely to be inconsistent with any other explanation that postulates that the old distractors influence the salience of the target in a purely bottom-up fashion.

Furthermore, it is also worth pointing out that the significant interaction observed between the number of old elements and the number of new elements in the present experiment is inconsistent not only with the attentional constraint hypothesis, but also with other recent findings that were obtained within the context of a low-salience search task. In particular, Theeuwes et al. (1998) also independently varied the number of old and new elements within the context of a search task in which the target appeared relatively nonsalient and showed that the efficiency of gap search was unaffected by the number of old distractors. Rather, in their experiment, gap-search times systematically increased as the number of new elements increased, regardless of how many old elements appeared in the preview display. Moreover, as was expected, the search rates observed in the gap condition were found to be approximately twice as efficient as the search rates in the all-element baseline condition and equally as efficient

Table 6

Slopes of the Display-Size Functions in Experiment 4 Listed as a Function of Search Type, Target Presence, and the Number of Old Elements (With $R^{2}$ )

\begin{tabular}{|c|c|c|c|c|c|c|c|c|}
\hline \multirow[b]{3}{*}{ Condition } & \multicolumn{8}{|c|}{ Old Elements } \\
\hline & \multicolumn{2}{|l|}{0} & \multicolumn{2}{|l|}{2} & \multicolumn{2}{|l|}{4} & \multicolumn{2}{|c|}{8} \\
\hline & Average & $\overline{R^{2}}$ & Average & $\overline{R^{2}}$ & Average & $R^{2}$ & Average & $R^{2}$ \\
\hline \multicolumn{9}{|l|}{ Gap } \\
\hline \multicolumn{9}{|c|}{ Target Absent } \\
\hline & & & 6.03 & 1.00 & 11.20 & 1.00 & 20.13 & 0.96 \\
\hline \multicolumn{9}{|c|}{ Target Present } \\
\hline & & & 4.24 & 1.00 & 7.03 & 1.00 & 11.48 & 0.99 \\
\hline \multicolumn{9}{|l|}{ Half-element } \\
\hline \multicolumn{9}{|c|}{ Target Absent } \\
\hline & -2.41 & 1.00 & & & & & & \\
\hline \multicolumn{9}{|c|}{ Target Present } \\
\hline & 1.41 & 0.29 & & & & & & \\
\hline
\end{tabular}


as those found in the half-element baseline condition. The discrepancy observed between the present results and Theeuwes et al.'s results is therefore important, because it further emphasizes how the consequences of visual marking can vary across high-salience search tasks and low-salience search tasks. More important, with respect to the attentional constraint hypothesis, this discrepancy also indicates that observers do not simply treat high-salience search tasks as low-salience search tasks within the context of the gap paradigm (otherwise, similar results should have been obtained across the two experiments).

\section{GENERAL DISCUSSION}

The present study has consistently shown that old distractors affect the efficiency of visual search in the highsalience gap condition, but not in the low-salience gap condition. The major implication of these findings is that visual marking appears to be limited in its ability to increase the efficiency of visual search. In particular, the present findings suggest that visual marking can increase the efficiency of visual search by decreasing the size of the search set, but not by increasing the perceptual salience of the target. Hence, the present findings suggest that the effectiveness of visual marking may depend on search context, with visual marking being more effective in lowsalience search contexts than in high-salience search contexts.

However, although the present findings can be interpreted to suggest that old distractors did affect the perceptual salience of the target in the high-salience gap condition, they were nevertheless unable to reveal exactly how this occurred. Two general types of processing constraints were considered in the present study. According to the perceptual constraint hypothesis, the old distractors were thought to decrease the salience of the target via purely bottom-up processing mechanisms, whereas according to the attentional constraint hypothesis, the old distractors were thought to decrease the salience of the target via purely top-down processing mechanisms. The failure to find supporting evidence for either one of these two theoretical extremes in Experiment 3 therefore suggests that the apparent influence of the old distractors on visual salience may not be due to any purely bottom-up or top-down processing constraints.

Of course, there are a variety of other possible constraints that could be fashioned around interactions between bottom-up and top-down mechanisms that may prove useful in future investigations. For instance, Enns and Di Lollo (1996) recently showed that an array of dots that had no effect on the perception of a visual target when attention was focused nevertheless had a devastating effect on the perception of the target when attention was divided across the display. Likewise, the perceptual influence of the old distractors may have varied as a function of attention in the present experiment, perhaps because attention became increasingly more divided as the number of new elements in the target display increased. The plausibility of this and other interactive accounts must await further evidence, however, and thus is beyond the scope of the present study.

Before closing, we would also like to briefly consider two alternative explanations of the decrease in search efficiency observed in the high-salience gap condition (relative to the high-salience half-element baseline condition). For instance, the old distractors may have affected search performance more in the high-salience gap condition than in the low-salience gap condition not because marking was less effective in high-salience search contexts, but rather because marking itself was applied less effectively to the old distractors in the high-salience gap condition. In the present study, we assumed that marking was applied equally to the old distractors in both the highsalience and the low-salience gap conditions for a number of reasons. First, the evidence obtained in Experiments 1 and 2 showed that search performance was approximately twice as efficient in the high-salience gap condition as in the corresponding all-element baseline condition, suggesting that only half the elements were searched.

In addition, high-salience gap trials were randomly mixed with low-salience gap trials in Experiment 1, and the findings obtained in the low-salience gap condition provided clear-cut evidence that the old distractors were successfully marked. Thus, we assumed that the old distractors were also successfully marked in the highsalience gap condition. However, this assumption may not be warranted, given that different preview displays preceded each of the two gap conditionsin Experiment 1. As a result, it is possible that the different pattern of results observed across the two gap conditions in Experiment 1 may have occurred because observers did not mark the old distractors as effectively in the high-salience gap condition as they did in the low-salience gap condition. Indeed, the task was easier in the high-salience gap condition than in the low-salience gap condition, and it may therefore have been possible to perform reasonably well in the former condition without expending as much energy on marking. However, this differential marking hypothesis appears to be inconsistent with the results of Experiment 3. In particular, as with the perceptual constraint hypothesis, the differential marking hypothesis also predicts that search through a given number of new elements should have become progressively slower as the number of old elements increased (because the old elements were not effectively ignored), but this result was not obtained in Experiment 3. Thus, it is unlikely that the pattern of results observed in the present study was obtained simply because observers varied the strength of visual marking across the two gap conditions.

Another important aspect of the present interpretation that should be reconsidered concerns the claim that the old distractors actually decreased the salience of the target in the high-salience gap condition. This conclusion was based on evidence obtained from all three experiments, which showed that search performance was less 
efficient in the high-salience gap condition than in the high-salience half-element baseline condition. In addition, the results of Experiment 3 were more specific and suggested that this decrease in search efficiency depended not only on the number of old elements, but also on the number of new elements. One alternative explanation of this decrease in search efficiency concerns the possibility that observers changed their response criteria as the overall display became more complex. In other words, the target may have appeared equally salient and thus may have been detected equally efficiently, regardless of the number of old and new elements that were shown in the high-salience gap condition, but observers may have simply demanded more evidence that the target was present when the display reached a certain critical level of complexity. As a result, the adoption of this more conservative criterion would have slowed search times and thus could possibility account for the unexpected pattern of results observed in Experiment 3.

We are currently investigating this criterion explanation of the difference in search efficiency observed between the high-salience gap condition and the highsalience half-element baseline condition within the context of a signal detection paradigm. Such a paradigm will allow us to determine whether the decrease in search efficiency observed in the high-salience gap condition reflects a change in criterion or a change in perceptual sensitivity. At this point in time, we can only offer our own subjective impression that the appearance of the target is actually harder to see in the high-salience gap condition than it is in the high-salience half-element baseline condition, especially when the number of old and new elements is relatively large. That is, the old distractors do in fact appear to decrease the perceptual salience of the target. Thus, we expect to find a change in perceptual sensitivity in the signal detection paradigm, and not merely a change in criterion.

Thus, the major conclusion of the present article is that visual marking can increase the efficiency of visual search by decreasing the size of the search set, but it is apparently limited in its ability to increase the efficiency of visual search by increasing the salience of the target. Although this conclusion may be judged to be inadequate with the benefit of additional evidence, there does not appear to be any strong reason to doubt its adequacy at the present point in time. Thus, the primary challenge for future investigationsinto the role of visual marking in high-salience search tasks will be the development of other perceptually based explanations that are capable of explaining why search efficiency depends on both the number of old elements and the number of new elements.

\section{REFERENCES}

Braun, J. (1998). Divided attention: Narrowing the gap between brain and behavior. In R. Parasuraman (Ed.), The attentive brain (pp. 327352). Cambridge, MA: MIT Press.

Desimone, R., \& Duncan, J. (1995). Neural mechanisms of selective visual attention. Annual Review of Neuroscience, 18, 193-222.
Desimone, R., Schein, S. G., Moran, J., \& Ungerleider, L. G. (1985). Contour, color and shape analysis beyond the striate cortex. Vision Research, 25, 441-452.

Duncan, J., \& Humphreys, G. W. (1989). Visual search and stimulus similarity. Psychological Review, 96, 433-458.

D’Zmura, M. (1991). Color in visual search. Vision Research, 31, 951966.

ENNS, J., \& Di Lollo, V. (1996). Object substitution: A new form of masking in unattended visual locations. Psychological Science, 8, 135-139.

GiBSON, B. S., \& JiANG, Y. (1998). Surprise! An unexpected color singleton does not capture attention in visual search. Psychological Science, 9, 176-182.

Horowitz, T., \& Wolfe, J. M. (1998). Visual search has no memory. Nature, 394, 575-577.

Klein, R. M., \& TAYlor, T. (1994). Categories of cognitive inhibition with reference to inhibition. In D. Dagenbach \& T. H. Carr (Eds.), Inhibitory processes in attention, memory, and language (pp. 113-150). San Diego: Academic Press.

Knierim, J. J., \& VAN ESSEN, D. C. (1992). Neuronal responses to static texture patterns in area V1 of the alert Macaque monkey. Journal of Neurophysiology, 67, 961-980.

NaGy, A. L., \& SANchez, R. R. (1990). Critical color differences determined with a visual search task. Journal of the Optical Society of America A, 7, 1209-1217.

NAKAYAma, K., \& JoSEPH, J. S. (1998). Attention, pattern recognition, and pop-out in visual search. In R. Parasuraman (Ed.), The attentive brain (pp. 279-298). Cambridge, MA: MIT Press.

Theeuwes, J. (1990). Perceptual selectivity is task dependent: Evidence from selective search. Acta Psychologica, 74, 81-99.

Theeuwes, J. (1991). Cross-dimensional perceptual selectivity. Perception \& Psychophysics, 50, 184-193.

Theeuwes, J. (1992). Perceptual selectivity for color and form. Perception \& Psychophysics, 51, 599-606.

Theeuwes, J., Kramer, A. F., \& Atchley, P. (1998). Visual marking of old objects. Psychonomic Bulletin \& Review, 5, 130-134.

Treisman, A. (1988). Features and objects: The fourteenth Bartlett memorial lecture. Quarterly Journal of Experimental Psychology, 40A, 401-437.

Treisman, A. (1994). The perception of features and objects. In A. Baddeley \& L. Weiskrantz (Eds.), Attention: Selection, awareness, and control (pp. 5-35). Oxford: Oxford University Press.

Treisman, A., \& Gelade, G. (1980). A feature integration theory of attention. Cognitive Psychology, 12, 97-136.

Watson, D. G., \& Humphreys, G. W. (1997). Visual marking: Prioritizing selection for new objects by top-down attentional inhibition of old objects. Psychological Review, 104, 90-122.

Wolfe, J. M. (1994). Guided Search 2.0: A revised model of visual search. Psychonomic Bulletin \& Review, 1, 202-238.

Wolfe, J. M. (1998). Visual search. In H. Pashler (Ed.), Attention (pp. 13-74). Hove, U.K.: Psychology Press.

\section{NOTES}

1. Display size was actually 2,4 , and 8 in the half-element baseline condition and 4, 8, and 16 in the all-element baseline and gap conditions. Nevertheless, in order to directly compare search efficiency in the half-element baseline condition with search efficiency in the other two conditions, all the slopes of the display-size functions were based on display sizes of 4, 8, and 16 (see Watson \& Humphreys, 1997, for a fuller discussion of this issue).

2. Note, however, that the findings reported in Experiment $4 \mathrm{~b}$ of Watson and Humphrey's (1997) study may provide weak evidence that the perceptual salience of the target can be increased in the gap condition. In this experiment, the target was a blue $H$; the distractors were blue $T \mathrm{~s}$ in the half-element baseline condition, and this display was added to a display of green $\mathrm{Hs}$ in the gap condition. Unlike in their other halfelement baseline condition, in which the blue $H$ target did not differ from the blue $A$ distractors along a primitive feature dimension, it is likely that the blue $H$ target could be distinguished from the blue $T$ distractors solely on the basis of orientation features. Thus, it is likely that 
the target did appear salient in the half-element baseline condition and had the potential to appear salient in the gap condition in this experiment. The results showed that search was relatively eff icient in the halfelement baseline condition (with RTs increasing by approximately $10 \mathrm{msec} / \mathrm{item}$ ). More important, the results of this experiment appeared to show that the blue $H$ target could be detected just as efficiently in the gap condition as in the half-element baseline condition, at least on target-present trials. However, the search latencies obtained in the gap condition of this experiment may have been speeded at the expense of accuracy, although an omnibus analysis of variance failed to reveal a significant interaction. For instance, mean percentage error rates on target-present trials in the gap condition of Experiment $4 \mathrm{~b}$ (Watson \& Humphreys, 1997; see Table 10) were $2.08 \%, 2.92 \%$, and $7.92 \%$ in the 4,8 , and 16 display-size conditions, respectively. In contrast, mean percentage error rates on target-present trials in the half-element baseline condition of Experiment 4b were 4.58\%, 3.33\%, 3.33\% in the 4, 8, and 16 display-size conditions, respectively. Hence, it is possible that Watson and Humphreys failed to find an RT difference between the gap and the half-element baseline conditions in this experiment because observers traded speed for accuracy in the gap condition on target-present trials. In addition, although their findings failed to reveal a significant difference in search efficiency across the gap and half-element baseline conditions on target-present trials, their findings did reveal a significant difference in search efficiency across the gap and half-element baseline conditions on target-absent trials. Thus, stronger evidence must be obtained to determine whether visual marking can increase the perceptual salience of the target.

3. We actually ran two versions of this experiment. One version was exactly as described in the text, and the other version was identical, except that the target was a blue $A$ and the distractors were blue $H$ s and green $A$ s. Subjects were randomly assigned to each of these two target conditions. For the sake of simplicity, we ignored this manipulation in the text, because the results were essentially identical in both groups.

(Manuscript received December 21, 1998; revision accepted for publication December 22, 1999.) 